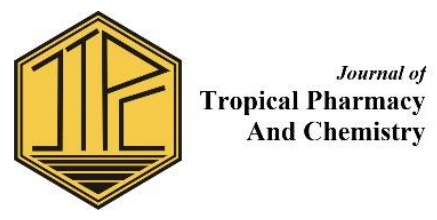

\title{
REVIEW: ANTIBACTERIAL ACTIVITIES OF VARIOUS PARTS OF MENGKUDU (Morinda citrifolia L.) PLANTS ON SOME SPECIES OF BACTERIA
}

\author{
Barolym Tri Pamungkas, Moelyono Muktiwardojo, Tina Rostinawati
}

Departement Biology Pharmacy, Faculty of Pharmacy, Padjadjaran University

Jl. Raya Bandung-Sumedang KM 21, Jatinangor 45363

Telephone (022) 7796200, Fax (022) 7796200

email: barolym@rocketmail.com

\begin{abstract}
The high incidence of infections and the increase in antibiotic resistance have become a global health concern. The emergence of strains of resistant bacteria has also encouraged the exploration of potential antibacterial agents, especially from natural sources. This review aims to provide information regarding the antibacterial activity of stems, leaves, fruits and seeds of mengkudu (M. citrifolia L,) in inhibiting various bacterial species. In this article review, online and offline literature were used. Online literature was obtained from local and international journals and scientific articles of the last 10 years, 2008-2018, which were obtained through direct search results online using online search engines namely Google and Google Scholar. Whereas the offline literature used is books and e-books. It is known that the root, stem, leaves, fruit, and seeds of mengkudu (M. citrifolia L,) with various solvents used can inhibit various species of bacteria with different diameter inhibition zones.
\end{abstract}

Keywords: $\quad$ M. citrifolia L, antibacterial, solvents

Submitted on: 11 January 2019

Accepted on: 03 June 2019

DOI: https://doi.org/10.25026/jtpc.v4i5.207

\section{INTRODUCTION}

The high incidence of infections and the increase in antibiotic resistance caused by overuse and misuse of antibiotics, improper administration, and limited availability of new generation antibiotics are becoming a global health concern. In addition, the emergence of resistant bacterial strains has also encouraged the exploration of potential antibacterial agents, especially from natural sources. ${ }^{1}$

The use of natural materials as medicine is the right thing that can support public health because it has minimal side effects when compared with drugs from chemicals. Medicinal plants play an important role in the treatment of several diseases, especially in developing countries. Many medicinal plants have been documented in pharmacopeia for 
herbal medicines because of their efficacy against pathogenic microbes. ${ }^{2}$

One of the plants that have been used by the community as a natural medicine ingredient is Morinda citrifolia L., commonly known as Mengkudu. Mengkudu is a plant that has almost all parts of its body as a medicine for both the stems, leaves, fruits, and seeds. This is because Mengkudu contains a lot of secondary metabolites which have many pharmacological activities in dealing with various diseases. Secondary metabolite compounds have polyvalent activity so that they can overcome various diseases. ${ }^{3}$ Therefore, the authors are interested in conducting a review of mengkudu. This review aims to provide information regarding the antibacterial activity of stem, leaves, fruit, and seeds of mengkudu ( $M$. citrifolia L,) in inhibiting various bacterial species.

\section{RESEARCH METHODS}

The data presented in this article, obtained from the literature online and offline. Online literature is obtained from local and international journals and scientific articles of the last 10 years, 20082018, which are obtained through direct search results online using online search engines namely Google and Google Scholar. Whereas the offline literature used is books and e-books.

\section{DISCUSSION}

Based on the search in several scientific journals, the authors found that various parts of Mengkudu (M. citrifoilia L.) plants can inhibit various species of bacteria, can be seen in Table 1 .

Table 1. 'Antibacterial activity of stem, leaves, fruit, and seeds of mengkudu (M. citrifoilia

L.) against various bacterial species (in $\mathrm{mm}$ ).

\begin{tabular}{|c|c|c|c|c|c|c|}
\hline $\begin{array}{c}\text { Parts of } \\
\text { plants used }\end{array}$ & Extract & Species & $\begin{array}{c}\text { The diameter } \\
\text { of the } \\
\text { inhibition } \\
\text { zone (mm) }\end{array}$ & Concentration & Method & Ref \\
\hline \multirow[t]{5}{*}{ Stem } & $\begin{array}{l}\text { Ethyl } \\
\text { acetate }\end{array}$ & $\begin{array}{ll}\text { 1. } & \text { Staphylococcus aureus } \\
\text { 2. } & \text { Streptococcus sp. } \\
\text { 3. } & \text { Proteus mirabilis } \\
\text { 4. } & \text { Pseudomonas diminuta } \\
\text { 5. } & \text { Enterobacter cloacae }\end{array}$ & $\begin{array}{l}6 \\
6 \\
6 \\
7 \\
6\end{array}$ & $\begin{array}{l}1000 \mu \mathrm{g} / \mathrm{mL} \\
1000 \mu \mathrm{g} / \mathrm{mL} \\
1000 \mu \mathrm{g} / \mathrm{mL} \\
1000 \mu \mathrm{g} / \mathrm{mL} \\
1000 \mu \mathrm{g} / \mathrm{mL}\end{array}$ & $\begin{array}{c}\text { Disc } \\
\text { diffusion }\end{array}$ & 4 \\
\hline & $\begin{array}{l}\text { Petroleum } \\
\text { ether }\end{array}$ & $\begin{array}{l}\text { 1. } \quad \text { Escherichia coli } \\
\text { 2. } \quad \text { Bacillus subtilis } \\
\text { 3. }\end{array}$ & $\begin{array}{l}17 \\
19 \\
20 \\
21 \\
17 \\
18\end{array}$ & $\begin{array}{c}5 \mathrm{mg} / \mathrm{mL} \\
10 \mathrm{mg} / \mathrm{mL} \\
5 \mathrm{mg} / \mathrm{mL} \\
10 \mathrm{mg} / \mathrm{mL} \\
5 \mathrm{mg} / \mathrm{mL} \\
10 \mathrm{mg} / \mathrm{mL}\end{array}$ & \multirow{2}{*}{$\begin{array}{c}\text { Disc } \\
\text { diffusion }\end{array}$} & \multirow{2}{*}{8} \\
\hline & \multirow[t]{2}{*}{ Ethanol } & $\begin{array}{l}\text { 1. Escherichia coli } \\
\text { 2. Bacillus subtilis } \\
\text { 3. Staphylococcus aureus }\end{array}$ & $\begin{array}{l}17 \\
19 \\
20 \\
21 \\
17 \\
18\end{array}$ & $\begin{array}{c}5 \mathrm{mg} / \mathrm{mL} \\
10 \mathrm{mg} / \mathrm{mL} \\
5 \mathrm{mg} / \mathrm{mL} \\
10 \mathrm{mg} / \mathrm{mL} \\
5 \mathrm{mg} / \mathrm{mL} \\
10 \mathrm{mg} / \mathrm{mL}\end{array}$ & & \\
\hline & & $\begin{array}{ll}\text { 1. } & \text { Staphylococcus aureus } \\
\text { 2. } & \text { Streptococcus sp. } \\
\text { 3. } & \text { Shigella flexneri } \\
\text { 4. } & \text { Pseudomonas diminuta } \\
\text { 5. } & \text { Enterobacter cloacae }\end{array}$ & $\begin{array}{c}6 \\
6 \\
6 \\
10 \\
6\end{array}$ & $\begin{array}{l}1000 \mu \mathrm{g} / \mathrm{mL} \\
1000 \mu \mathrm{g} / \mathrm{mL} \\
1000 \mu \mathrm{g} / \mathrm{mL} \\
1000 \mu \mathrm{g} / \mathrm{mL} \\
1000 \mu \mathrm{g} / \mathrm{mL}\end{array}$ & $\begin{array}{c}\text { Disc } \\
\text { diffusion }\end{array}$ & 4 \\
\hline & Methanol & $\begin{array}{l}\text { 1. Escherichia coli } \\
\text { 2. Pseudomonas aeruginosa } \\
\text { 3. }\end{array}$ & $\begin{array}{l}7 \\
6 \\
6\end{array}$ & $\begin{array}{l}1000 \mu \mathrm{g} / \mathrm{mL} \\
1000 \mu \mathrm{g} / \mathrm{mL} \\
1000 \mu \mathrm{g} / \mathrm{mL}\end{array}$ & $\begin{array}{c}\text { Disc } \\
\text { diffusion }\end{array}$ & 4 \\
\hline
\end{tabular}




\begin{tabular}{|c|c|c|c|c|c|c|}
\hline & & $\begin{array}{ll}\text { 4. } & \text { Streptococcus sp. } \\
\text { 5. } & \text { Shigella flexneri } \\
\text { 6. } & \text { Proteus mirabilis } \\
\text { 7. } & \text { Pseudomonas diminuta } \\
\text { 8. } & \text { Enterobacter cloacae } \\
\end{array}$ & $\begin{array}{l}8 \\
6 \\
6 \\
6 \\
6\end{array}$ & $\begin{array}{l}1000 \mu \mathrm{g} / \mathrm{mL} \\
1000 \mu \mathrm{g} / \mathrm{mL} \\
1000 \mu \mathrm{g} / \mathrm{mL} \\
1000 \mu \mathrm{g} / \mathrm{mL} \\
1000 \mu \mathrm{g} / \mathrm{mL}\end{array}$ & & \\
\hline \multirow[t]{7}{*}{ Leaf } & Methanol & $\begin{array}{ll}\text { 1. } & \text { Escherichia coli } \\
\text { 2. } & \text { Pseudomonas sp. } \\
\text { 3. } & \text { Salmonella sp. } \\
\text { 4. } & \text { Staphylococcus aureus } \\
\text { 5. } & \text { Klebsiella sp. }\end{array}$ & $\begin{array}{c}10,8 \\
8,8 \\
12,6 \\
11,6 \\
6,2 \\
\end{array}$ & $\begin{array}{l}5 \mathrm{mg} / \mathrm{mL} \\
5 \mathrm{mg} / \mathrm{mL} \\
5 \mathrm{mg} / \mathrm{mL} \\
5 \mathrm{mg} / \mathrm{mL} \\
5 \mathrm{mg} / \mathrm{mL}\end{array}$ & $\begin{array}{c}\text { Disc } \\
\text { diffusion }\end{array}$ & \multirow{5}{*}{$4,5,6$} \\
\hline & & $\begin{array}{ll}\text { 1. } & \text { Escherichia coli } \\
\text { 2. } & \text { Pseudomonas aeruginosa } \\
\text { 3. } & \text { Staphylococcus aureus } \\
\text { 4. } & \text { Klebsiella pneumonia } \\
\text { 5. } & \text { Streptococcus sp. } \\
\text { 6. } & \text { Shigella flexneri } \\
\text { 7. } & \text { Proteus mirabilis } \\
\text { 8. } & \text { Pseudomonas diminuta } \\
\text { 9. } & \text { Pseudomonas fluorescens } \\
\text { 10. } & \text { Enterobacter cloacae }\end{array}$ & $\begin{array}{c}8 \\
6 \\
9 \\
7 \\
6 \\
8 \\
6 \\
11 \\
8 \\
7 \\
\end{array}$ & $\begin{array}{l}1000 \mu \mathrm{g} / \mathrm{mL} \\
1000 \mu \mathrm{g} / \mathrm{mL} \\
1000 \mu \mathrm{g} / \mathrm{mL} \\
1000 \mu \mathrm{g} / \mathrm{mL} \\
1000 \mu \mathrm{g} / \mathrm{mL} \\
1000 \mu \mathrm{g} / \mathrm{mL} \\
1000 \mu \mathrm{g} / \mathrm{mL} \\
1000 \mu \mathrm{g} / \mathrm{mL} \\
1000 \mu \mathrm{g} / \mathrm{mL} \\
1000 \mu \mathrm{g} / \mathrm{mL}\end{array}$ & $\begin{array}{c}\text { Disc } \\
\text { diffusion }\end{array}$ & \\
\hline & Chloroform & $\begin{array}{ll}\text { 1. } & \text { Escherichia coli } \\
\text { 2. } & \text { Pseudomonas sp. } \\
\text { 3. } & \text { Salmonella sp. } \\
\text { 4. } & \text { Staphylococcus aureus } \\
\text { 5. } & \text { Klebsiella sp. } \\
\end{array}$ & $\begin{array}{c}8,8 \\
8,4 \\
11,6 \\
11,0 \\
8,8 \\
\end{array}$ & $\begin{array}{l}5 \mathrm{mg} / \mathrm{mL} \\
5 \mathrm{mg} / \mathrm{mL} \\
5 \mathrm{mg} / \mathrm{mL} \\
5 \mathrm{mg} / \mathrm{mL} \\
5 \mathrm{mg} / \mathrm{mL}\end{array}$ & $\begin{array}{c}\text { Disc } \\
\text { diffusion }\end{array}$ & \\
\hline & Acetone & $\begin{array}{ll}\text { 1. } & \text { Escherichia coli } \\
\text { 2. } & \text { Pseudomonas sp. } \\
\text { 3. } & \text { Salmonella sp. } \\
\text { 4. } & \text { Staphylococcus aureus } \\
\text { 5. } & \text { Klebsiella sp. }\end{array}$ & $\begin{array}{c}14,2 \\
11 \\
13,2 \\
11,2 \\
6,211,2 \\
\end{array}$ & $\begin{array}{l}5 \mathrm{mg} / \mathrm{mL} \\
5 \mathrm{mg} / \mathrm{mL} \\
5 \mathrm{mg} / \mathrm{mL} \\
5 \mathrm{mg} / \mathrm{mL} \\
5 \mathrm{mg} / \mathrm{mL}\end{array}$ & $\begin{array}{c}\text { Disc } \\
\text { diffusion }\end{array}$ & \\
\hline & $\begin{array}{l}\text { Hydro- } \\
\text { alcohol }\end{array}$ & $\begin{array}{l}\text { 1. Bacillus subtilis } \\
\text { 2. Pseudomonas aeruginosa } \\
\text { 3. Staphylococcus aureus } \\
\text { 4. Proteus vulgaris } \\
\text { 5. Eschericia coli } \\
\text { 6. Serratia marcescens }\end{array}$ & $\begin{array}{c}7 \\
11 \\
10 \\
11 \\
11 \\
11 \\
12 \\
10 \\
11 \\
- \\
6 \\
6 \\
6 \\
7 \\
7 \\
7 \\
8 \\
8 \\
\end{array}$ & $\begin{array}{c}5 \mu \mathrm{L} \\
10 \mu \mathrm{L} \\
20 \mu \mathrm{L} \\
5 \mu \mathrm{L} \\
10 \mu \mathrm{L} \\
20 \mu \mathrm{L} \\
5 \mu \mathrm{L} \\
10 \mu \mathrm{L} \\
20 \mu \mathrm{L} \\
5 \mu \mathrm{L} \\
10 \mu \mathrm{L} \\
20 \mu \mathrm{L} \\
5 \mu \mathrm{L} \\
10 \mu \mathrm{L} \\
20 \mu \mathrm{L} \\
5 \mu \mathrm{L} \\
10 \mu \mathrm{L} \\
20 \mu \mathrm{L}\end{array}$ & $\begin{array}{c}\text { Disc } \\
\text { diffusion }\end{array}$ & \\
\hline & Ethanol & $\begin{array}{ll}\text { 1. } & \text { Escherichia coli } \\
\text { 2. } & \text { Staphylococcus aureus } \\
\text { 3. } & \text { Klebsiella pneumonia } \\
\text { 4. } & \text { Shigella flexneri } \\
\text { 5. } & \text { Proteus mirabilis } \\
\text { 6. } & \text { Pseudomonas diminuta } \\
\text { 7. } & \text { Pseudomonas fluorescens } \\
\text { 8. } & \text { Enterobacter cloacae } \\
\end{array}$ & $\begin{array}{c}7 \\
8 \\
6 \\
8 \\
6 \\
10 \\
6 \\
6 \\
\end{array}$ & $\begin{array}{l}1000 \mu \mathrm{g} / \mathrm{mL} \\
1000 \mu \mathrm{g} / \mathrm{mL} \\
1000 \mu \mathrm{g} / \mathrm{mL} \\
1000 \mu \mathrm{g} / \mathrm{mL} \\
1000 \mu \mathrm{g} / \mathrm{mL} \\
1000 \mu \mathrm{g} / \mathrm{mL} \\
1000 \mu \mathrm{g} / \mathrm{mL} \\
1000 \mu \mathrm{g} / \mathrm{mL}\end{array}$ & $\begin{array}{c}\text { Disc } \\
\text { diffusion }\end{array}$ & 4 \\
\hline & $\begin{array}{c}\text { Ethyl } \\
\text { acetate }\end{array}$ & $\begin{array}{ll}\text { 1. } & \text { Escherichia coli } \\
\text { 2. } & \text { Staphylococcus aureus } \\
\text { 3. } & \text { Klebsiella pneumonia } \\
\text { 4. } & \text { Streptococcus sp. } \\
\text { 5. } & \text { Shigella flexneri } \\
\text { 6. } & \text { Proteus mirabilis } \\
\text { 7. } & \text { Pseudomonas diminuta } \\
\text { 8. } & \text { Pseudomonas fluorescens }\end{array}$ & $\begin{array}{l}6 \\
7 \\
6 \\
6 \\
7 \\
6 \\
9 \\
7\end{array}$ & $\begin{array}{l}1000 \mu \mathrm{g} / \mathrm{mL} \\
1000 \mu \mathrm{g} / \mathrm{mL} \\
1000 \mu \mathrm{g} / \mathrm{mL} \\
1000 \mu \mathrm{g} / \mathrm{mL} \\
1000 \mu \mathrm{g} / \mathrm{mL} \\
1000 \mu \mathrm{g} / \mathrm{mL} \\
1000 \mu \mathrm{g} / \mathrm{mL} \\
1000 \mu \mathrm{g} / \mathrm{mL}\end{array}$ & $\begin{array}{c}\text { Disc } \\
\text { diffusion }\end{array}$ & 4 \\
\hline
\end{tabular}




\begin{tabular}{|c|c|c|c|c|c|c|}
\hline \multirow[t]{6}{*}{ Fruit } & \multirow[t]{2}{*}{ Methanol } & $\begin{array}{l}\text { 1. } \text { Bacillus subtilis } \\
\text { 2. } \text { Staphylococcus aureus } \\
\text { 3. Lactobacillus lactis } \\
\text { 4. } \text { Streptococcus thermophilus } \\
\text { 5. Pseudomonas aeruginosa } \\
\text { 6. Salmonella typhi } \\
\text { 7. Eschericia coli } \\
\text { 8. Vibrio harveyi } \\
\text { 9. Klebsiella pneumonia } \\
\text { 10. Shigella flexneri } \\
\text { 11. Salmonella paratyphi } \\
\text { 12. Aeromonas hydrophila } \\
\text { 13. Vibrio cholera } \\
\text { 14. Chromobacterium Violaceum } \\
\text { 15. Enterobacter faecalis }\end{array}$ & $\begin{array}{c}10 \\
11,3 \\
10,0 \\
11,3 \\
10,3 \\
10,3 \\
11,0 \\
11,3 \\
9,3 \\
11,0 \\
26,0 \\
12,3 \\
7,7 \\
12,3 \\
13,0\end{array}$ & $\begin{array}{l}100 \mathrm{mg} / \mathrm{mL} \\
100 \mathrm{mg} / \mathrm{mL} \\
100 \mathrm{mg} / \mathrm{mL} \\
100 \mathrm{mg} / \mathrm{mL} \\
100 \mathrm{mg} / \mathrm{mL} \\
100 \mathrm{mg} / \mathrm{mL} \\
100 \mathrm{mg} / \mathrm{mL} \\
100 \mathrm{mg} / \mathrm{mL} \\
100 \mathrm{mg} / \mathrm{mL} \\
100 \mathrm{mg} / \mathrm{mL} \\
100 \mathrm{mg} / \mathrm{mL} \\
100 \mathrm{mg} / \mathrm{mL} \\
100 \mathrm{mg} / \mathrm{mL} \\
100 \mathrm{mg} / \mathrm{mL} \\
100 \mathrm{mg} / \mathrm{mL}\end{array}$ & $\begin{array}{c}\text { Cup } \\
\text { Plate } \\
\text { Tehnique }\end{array}$ & 7 \\
\hline & & $\begin{array}{ll}\text { 1. } & \text { Escherichia coli } \\
\text { 2. } & \text { Pseudomonas aeruginosa } \\
\text { 3. } & \text { Staphylococcus aureus } \\
\text { 4. } & \text { Klebsiella pneumonia } \\
\text { 5. } & \text { Streptococcus sp. } \\
\text { 6. } & \text { Shigella flexneri } \\
\text { 7. } & \text { Proteus mirabilis } \\
\text { 8. } & \text { Pseudomonas diminuta } \\
\text { 9. } & \text { Pseudomonas fluorescens } \\
\text { 10. } & \text { Enterobacter cloacae }\end{array}$ & $\begin{array}{l}7 \\
7 \\
7 \\
8 \\
6 \\
7 \\
8 \\
8 \\
6 \\
8\end{array}$ & $\begin{array}{l}1000 \mu \mathrm{g} / \mathrm{mL} \\
1000 \mu \mathrm{g} / \mathrm{mL} \\
1000 \mu \mathrm{g} / \mathrm{mL} \\
1000 \mu \mathrm{g} / \mathrm{mL} \\
1000 \mu \mathrm{g} / \mathrm{mL} \\
1000 \mu \mathrm{g} / \mathrm{mL} \\
1000 \mu \mathrm{g} / \mathrm{mL} \\
1000 \mu \mathrm{g} / \mathrm{mL} \\
1000 \mu \mathrm{g} / \mathrm{mL} \\
1000 \mu \mathrm{g} / \mathrm{mL}\end{array}$ & $\begin{array}{c}\text { Disc } \\
\text { diffusion }\end{array}$ & 5 \\
\hline & Ethanol & $\begin{array}{ll}\text { 1. } & \text { Escherichia coli } \\
\text { 2. } & \text { Pseudomonas aeruginosa } \\
\text { 3. } & \text { Staphylococcus aureus } \\
\text { 4. } & \text { Streptococcus sp. } \\
\text { 5. } & \text { Shigella flexneri } \\
\text { 6. } & \text { Proteus mirabilis } \\
\text { 7. } & \text { Pseudomonas diminuta } \\
\text { 8. } & \text { Pseudomonas fluorescens } \\
\text { 9. } & \text { Enterobacter cloacae }\end{array}$ & $\begin{array}{l}6 \\
7 \\
8 \\
8 \\
9 \\
6 \\
7 \\
6 \\
6\end{array}$ & $\begin{array}{l}1000 \mu \mathrm{g} / \mathrm{mL} \\
1000 \mu \mathrm{g} / \mathrm{mL} \\
1000 \mu \mathrm{g} / \mathrm{mL} \\
1000 \mu \mathrm{g} / \mathrm{mL} \\
1000 \mu \mathrm{g} / \mathrm{mL} \\
1000 \mu \mathrm{g} / \mathrm{mL} \\
1000 \mu \mathrm{g} / \mathrm{mL} \\
1000 \mu \mathrm{g} / \mathrm{mL} \\
1000 \mu \mathrm{g} / \mathrm{mL}\end{array}$ & $\begin{array}{c}\text { Disc } \\
\text { diffusion }\end{array}$ & 4 \\
\hline & \multirow[t]{2}{*}{$\begin{array}{l}\text { Ethyl } \\
\text { acetate }\end{array}$} & 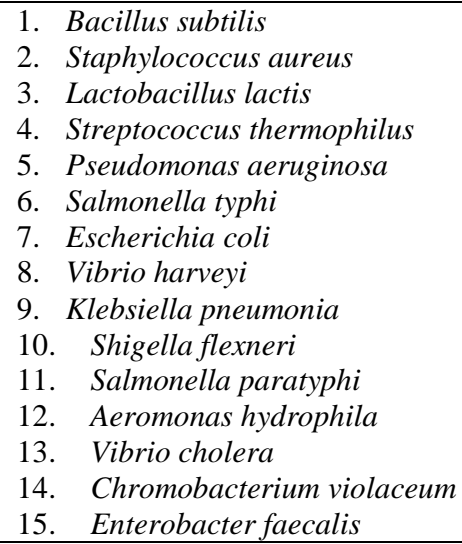 & $\begin{array}{c}6,3 \\
6,7 \\
5,7 \\
6,0 \\
- \\
6,0 \\
6,3 \\
6,3 \\
- \\
8,0 \\
15,7 \\
11,3 \\
10,7 \\
12,0 \\
14,7\end{array}$ & $\begin{array}{l}100 \mathrm{mg} / \mathrm{mL} \\
100 \mathrm{mg} / \mathrm{mL} \\
100 \mathrm{mg} / \mathrm{mL} \\
100 \mathrm{mg} / \mathrm{mL} \\
100 \mathrm{mg} / \mathrm{mL} \\
100 \mathrm{mg} / \mathrm{mL} \\
100 \mathrm{mg} / \mathrm{mL} \\
100 \mathrm{mg} / \mathrm{mL} \\
100 \mathrm{mg} / \mathrm{mL} \\
100 \mathrm{mg} / \mathrm{mL} \\
100 \mathrm{mg} / \mathrm{mL} \\
100 \mathrm{mg} / \mathrm{mL} \\
100 \mathrm{mg} / \mathrm{mL} \\
100 \mathrm{mg} / \mathrm{mL} \\
100 \mathrm{mg} / \mathrm{mL}\end{array}$ & $\begin{array}{c}\text { Cup } \\
\text { Plate } \\
\text { Tehnique }\end{array}$ & 7 \\
\hline & & $\begin{array}{ll}\text { 1. } & \text { Escherichia coli } \\
\text { 2. } & \text { Klebsiella pneumonia } \\
\text { 3. } & \text { Shigella flexneri } \\
\text { 4. } & \text { Enterobacter cloacae }\end{array}$ & $\begin{array}{l}6 \\
6 \\
7 \\
6\end{array}$ & $\begin{array}{l}1000 \mu \mathrm{g} / \mathrm{mL} \\
1000 \mu \mathrm{g} / \mathrm{mL} \\
1000 \mu \mathrm{g} / \mathrm{mL} \\
1000 \mu \mathrm{g} / \mathrm{mL}\end{array}$ & $\begin{array}{c}\text { Disc } \\
\text { diffusion }\end{array}$ & 4 \\
\hline & n-hexan & $\begin{array}{ll}\text { 1. } & \text { Bacillus subtilis } \\
\text { 2. } & \text { Staphylococcus aureus } \\
\text { 3. } & \text { Lactobacillus lactis } \\
\text { 4. } & \text { Streptococcus thermophilus } \\
\text { 5. } & \text { Pseudomonas aeruginosa } \\
\text { 6. } & \text { Salmonella typhi } \\
\text { 7. } & \text { Eschericia coli } \\
\text { 8. } & \text { Vibrio harveyi } \\
\text { 9. } & \text { Klebsiella pneumonia } \\
\text { 10. Shigella flexneri } \\
\text { 11. Salmonella paratyphi }\end{array}$ & $\begin{array}{l}- \\
- \\
6,3 \\
- \\
- \\
- \\
- \\
- \\
- \\
- \\
-\end{array}$ & $\begin{array}{l}100 \mathrm{mg} / \mathrm{mL} \\
100 \mathrm{mg} / \mathrm{mL} \\
100 \mathrm{mg} / \mathrm{mL} \\
100 \mathrm{mg} / \mathrm{mL} \\
100 \mathrm{mg} / \mathrm{mL} \\
100 \mathrm{mg} / \mathrm{mL} \\
100 \mathrm{mg} / \mathrm{mL} \\
100 \mathrm{mg} / \mathrm{mL} \\
100 \mathrm{mg} / \mathrm{mL} \\
100 \mathrm{mg} / \mathrm{mL} \\
100 \mathrm{mg} / \mathrm{mL}\end{array}$ & $\begin{array}{c}\text { Cup } \\
\text { Plate } \\
\text { Tehnique }\end{array}$ & 7 \\
\hline
\end{tabular}




\begin{tabular}{|c|c|c|c|c|c|c|}
\hline & & $\begin{array}{l}\text { 12. Aeromonas hydrophila } \\
\text { 13. Vibrio cholera } \\
\text { 14. Chromobacterium violaceum } \\
\text { 15. Enterobacter faecalis }\end{array}$ & $\begin{array}{l}- \\
- \\
- \\
-\end{array}$ & $\begin{array}{l}100 \mathrm{mg} / \mathrm{mL} \\
100 \mathrm{mg} / \mathrm{mL} \\
100 \mathrm{mg} / \mathrm{mL} \\
100 \mathrm{mg} / \mathrm{mL} \\
\end{array}$ & & \\
\hline \multirow[t]{3}{*}{ Seeds } & Methanol & $\begin{array}{ll}\text { 1. } & \text { Escherichia coli } \\
\text { 2. } & \text { Pseudomonas } s p . \\
\text { 3. } & \text { Salmonella } s p . \\
\text { 4. } & \text { Staphylococcus aureus } \\
\text { 5. } & \text { Klebsiella } s p . \\
\end{array}$ & $\begin{array}{c}12,8 \\
12,4 \\
12 \\
13,4 \\
12\end{array}$ & $\begin{array}{l}5 \mathrm{mg} / \mathrm{mL} \\
5 \mathrm{mg} / \mathrm{mL} \\
5 \mathrm{mg} / \mathrm{mL} \\
5 \mathrm{mg} / \mathrm{mL} \\
5 \mathrm{mg} / \mathrm{mL}\end{array}$ & $\begin{array}{c}\text { Disc } \\
\text { diffusion }\end{array}$ & \multirow{3}{*}{6} \\
\hline & Chloroform & $\begin{array}{ll}\text { 1. } & \text { Escherichia coli } \\
\text { 2. } & \text { Pseudomonas sp. } \\
\text { 3. } & \text { Salmonella sp. } \\
\text { 4. } & \text { Staphylococcus aureus } \\
\text { 5. } & \text { Klebsiella sp. }\end{array}$ & $\begin{array}{l}11,6 \\
12,6 \\
11,2 \\
13,0 \\
11,2\end{array}$ & $\begin{array}{l}5 \mathrm{mg} / \mathrm{mL} \\
5 \mathrm{mg} / \mathrm{mL} \\
5 \mathrm{mg} / \mathrm{mL} \\
5 \mathrm{mg} / \mathrm{mL} \\
5 \mathrm{mg} / \mathrm{mL}\end{array}$ & $\begin{array}{c}\text { Disc } \\
\text { diffusion }\end{array}$ & \\
\hline & Acetone & $\begin{array}{ll}\text { 1. } & \text { Escherichia coli } \\
\text { 2. } & \text { Pseudomonas } s p . \\
\text { 3. } & \text { Salmonella } s p . \\
\text { 4. } & \text { Staphylococcus aureus } \\
\text { 5. } & \text { Klebsiella } s p .\end{array}$ & $\begin{array}{l}11,8 \\
13,6 \\
12,2 \\
11,4 \\
12,2\end{array}$ & $\begin{array}{l}5 \mathrm{mg} / \mathrm{mL} \\
5 \mathrm{mg} / \mathrm{mL} \\
5 \mathrm{mg} / \mathrm{mL} \\
5 \mathrm{mg} / \mathrm{mL} \\
5 \mathrm{mg} / \mathrm{mL}\end{array}$ & $\begin{array}{c}\text { Disc } \\
\text { diffusion }\end{array}$ & \\
\hline
\end{tabular}

The extraction process is one of the first important steps in the preparation of plant formulations. Some researchers have made considerable efforts to find efficient extraction methods in order to obtain high efficiency and efficacy of course. ${ }^{9}$ The choice of the right solvent is very important because if the choice of solvent is not suitable, the results obtained are little or not obtained at all because the solvent is not appropriate. $^{10}$

Disc diffusion method is more widely used by some researchers because this method is quite accurate, economical, very easy to do and there are some researchers who advocate its use in routine clinical testing. ${ }^{11}$ In addition, other researchers have also succeeded in using the Disc diffusion method to test the susceptibility of Candida species to azole antifungal agents and various types of fungi such as Fusarium, Scedosporium, and other dematiaceous fungi. ${ }^{12}$

\section{CONCLUSION}

Natural resources are still considered as potential candidates for drug discovery and play an important role in drug development programs. Various parts of the noni fruit in the stem, leaves, fruit and noni seeds show antibacterial activity.
Some researchers believe that the presence of bioactive chemicals is primarily responsible for this antibacterial action. Further work is needed to isolate this active agent which can be used as a good source of antibiotics.

\section{REFERENCE}

[1] Ventola CL. 2015. The Antibiotic Resistance Crisis Part 1: Causes and Threats. Pharmacy and Therapeutics. 40(4):277-283.

[2] Palombo EA. 2011. Traditional Medicinal Plant Extracts and Natural Products With Activity Against Oral Bacteria: Potential Application In The Prevention And Treatment of Oral Diseases. Evidence-based Complementary and Alternative Medicine. 11: 680354.

[3] Bone, K., and Mills, S. 2013. Principles and Practice of Phytotherapy Second Edition. Churchill Livingstone Elsevier, New York.

[4] Natheer, S.E., C. Sekar, P. Amutharaj, M. Syed Abdul Rahman, and K. Feroz Khan. 2012. Evaluation of antibacterial activity of Morinda citrifolia, Vitex trifolia, and Chromolaena odorata. African 
Journal of Pharmacy and Pharmacology. 6(11): 783-788.

[5] Nayak, B.K., V. Suchitra, Anima Nanda. 2015. Antibacterial Potency of Hydro-Alcohol Leaf Extract of Morinda Citrifolia L. (Noni) by Soxhlet Extraction Method. Der Pharmacia Lettre, 7 (4):51-54.

[6] Sunder, J., D.R.Singh, S.Jeyakumar, A.Kundu, Arun Kumar De. 2011. Antibacterial Activity in Solvent Extract of Different Parts of Morinda citrifolia Plant. Journal of Pharmaceutical Sciences and Research. 3(8): 1404-1407.

[7] Jayaraman, S.K., Muthu S.M., Seethalakshmi, I. 2008. Antibacterial, Antifungal and Tumor cell suppression potential of Morinda citrifolia fruit extracts. International Journal of Integrative Biology. 3(1): 44-49.

[8] Devi, C.H.K and Gopala, K.D. 2013. Phytochemical Screening, Antibacterial, Antifungal and Anthelmintic Activity of Morinda citrifolia Stem. Journal of Pharmacognosy and Phytochemistry. 2(1): 115-117.
[9] Gupta A, Naraniwal M., Kothari V. 2012. Modern Extraction Methods for Preparation of Bioactive Plant Extracts. International Journal of Applied and Natural Sciences. 1(1): 8-26.

[10] Mujipradhana, V.N., Defny S. Wewengkang and Edi Suryanto. 2018. Aktivitas Antimikroba Dari Ekstrak Ascidian Herdmania momus Pada Mikroba Patogen Manusia. Pharmacon Jurnal Ilmiah Farmasi. 7(3): 2302 - 2493.

[11] Nweze, E.I., P. K. Mukherjee, M. A. Ghannoum. 2010. Agar-Based Disk Diffusion Assay for Susceptibility Testing of Dermatophytes. Journal of Clinical Microbiology. 48(10): 3750-3752.

[12] Bagul, U.S and Sivagurunathan M. Sivakumar. 2016. Antibiotic Susceptibility Testing: A Review of Current Practices. International Journal of Pharmacy. 6(3): 11-17. 